\title{
Thermal Analysis of Bubble Column Reactor Operated at Elevated Pressure
}

\author{
Mostafa H. Sharqawy \\ School of Engineering, University of Guelph \\ Guelph, Ontario N1G 2W1, Canada \\ melsharq@uoguelph.ca
}

\begin{abstract}
Bubble column reactors are well-known devices in many industrial applications for gas-liquid reactions. Recently, they are being used as humidifiers and dehumidifiers in desalination and water treatment systems because of their high effectiveness and low size compared with finned-tube heat exchangers. It was demonstrated that operating a bubble column reactor at a pressure higher than the atmospheric one, increases its thermal performance. However, these theoretical studies still lack experimental evidences. In this paper, the experimental performance of a bubble column reactor is presented in terms of the total heat transfer rate and the effectiveness. The experiments were performed under absolute pressure in the range of $1-2$ bars, superficial velocity in the range of $2-18 \mathrm{~cm} / \mathrm{s}$, and bubble column liquid height in the range of $3-7 \mathrm{~cm}$. The results show that the liquid water height in the bubble column has a negligible effect on both the total heat transfer and the effectiveness. However, it was found that the superficial velocity significantly increases the heat transfer rate and effectiveness. In addition, increasing the operating pressure inside the bubble column helps to slightly increase the heat transfer rate, but it leads to a decrease in the effectiveness.
\end{abstract}

Keywords: Bubble column, Pressure effect, Experimental.

\section{Introduction}

Bubble columns are devices where gas bubbles come in contact with liquid and a simultaneous direct heat and mass transfer processes occur. The gas is sparged from the bottom of the liquid using porous plates or perforated tubes. The main purpose of the bubble columns is to generate small gas bubbles to maximize the interfacial area between the liquid and gas phase. Having a large interfacial area increases the intensity of heat and mass transfer. Some investigators [1 - 4] proposed to use bubble column reactors as humidifiers and dehumidifiers in desalination systems. In these systems, cold-dry air is sparged into a column of hot water to be heated and humidified (i.e. bubble column humidifier), and hot-humid air is sparged into a column of cold water to condense the vapor carried by the air and produce fresh water (bubble column dehumidifier). Heat and mass transfer occur in the bubble column as long as there are temperature and concentration differences between the water vapor carried by the air bubbles and the liquid water in the bubble column. The transfer of the water molecules is determined by many parameters, including the gas holdup, bubble diameter, superficial gas velocity, pressure, and the fluids properties [5]. These aspects lead to extensive studies of system design and performance analysis of bubble columns. Bubble column plays an important role in the chemical processes simultaneously with transport phenomena and energy conversion occurring. They are being used in many industrial applications such as humidification process for desalination application, oxidation and chlorination in chemical industries and scrubbing of $\mathrm{CO} 2$ greenhouse gases.

It is well known that the heat transfer coefficient during film condensation of air-vapor mixture is very low because of the large mass presence of non-condensable gases (e.g. air). In bubble columns, the gas is dispersed into a pool of liquid to form bubbly flow instead of a direct contact with a solid surface. This results of a large interfacial area and low gas side resistance. Consequently, the overall performance of the heat and mass transfer processes is enhanced and an enormous saving in the device size ensued. These are considered as excellent advantages of bubble columns where the vapor carried by the air bubbles condenses on the interfacial area between the bubble surface and the cold liquid film in the column. The latent heat of vaporization is released from the air-vapor mixture to the water.

Narayan et al [3] developed an analytical model and presented experimental results for the performance of a bubble column dehumidifier. They proposed a thermal resistance model involved some remarkable thermal resistances associated 
with the different temperatures. The bubble column dehumidifier had dimensions of $30 \mathrm{~cm} \times 30 \mathrm{~cm} \times 46 \mathrm{~cm}$ and it was designed with an aluminium sparger located at its bottom to generate and distribute air bubbles. The cold source in the dehumidifier was provided by the coolant flow in a cooling coil made of copper and inserted inside the column. The heat flux was determined by varying operating conditions such as superficial velocity, bubble diameter and column liquid height. The results demonstrated that high superficial velocity and low bubble diameter are favourable to the heat flux, and the column liquid height above $15 \mathrm{~cm}$ has no effect on the heat flux.

A further investigation was carried out by Tow and Lienhard V [4] to measure the heat flux and parallel flow effectiveness of a bubble column dehumidifier for a superficial velocity range of 1.9-3.2 cm/s, a water height in the bubble column of 4-20 cm, and an air inlet temperature of $40-60{ }^{\circ} \mathrm{C}$. In addition, a simplified heat and mass transfer model was proposed by neglecting the gas side thermal resistance. Their theoretical and experimental results show that increasing both of the superficial velocity and inlet air temperature led to an increase in the heat flux and a decrease in the effectiveness. Moreover, the two design parameters of bubble column, water height and bubble on coil impact, demonstrated insignificant effect on the heat flux and effectiveness.

Bubble columns are usually operated at non-atmospheric conditions in industrial applications [6]. Although it was reported by Letzel et al [7] that the pressure has a significant effect on the hydrodynamics of bubble column reactors, studies [8 - 14] investigated the influence of pressure on the gas holdup, bubble size, and mass transfer coefficients in bubble column reactors. However, the effect of the operating pressure on bubble column dehumidifiers was not investigated. There are advantages of using variable pressure HDH desalination system as theoretically presented by Narayan et al [12]. This study suggested operating the dehumidifier of the HDH system at a pressure higher than the atmospheric one to increase the performance of the HDH system. However, this theoretical study still lacks experimental evidence to support it.

In the present work, the performance of a bubble column dehumidifier is experimentally investigated under elevated pressure for HDH desalination system. The total heat transfer rate and the effectiveness are measured at different operating pressures, superficial gas velocities, and liquid height in the bubble column.

\section{Experimental Work}

A laboratory scale bubble column dehumidifier is designed and built in order to measure its performance at different operating conditions. Hot-humid air is sparged from a perforated plate at the bottom of the bubble column and into a pool of cold water to form a bubbly flow. Water is circulated inside a cooling copper coil immersed in the bubble column and receives the heat released from the hot-humid air. The temperature difference between the air bubbles and the water in the bubble column contributes to the sensible cooling of the air. While the water vapor concentration (or humidity ratio) difference drives the latent cooling and the condensation occurs at the surface of the bubble.

A schematic and a photograph of the bubble column dehumidifier during the experimental test are shown in Fig. 1. The bubble column dehumidifier consists of a transparent PVC tube (100 mm ID, $250 \mathrm{~mm}$ height), a perforated plate made of stainless steel ( $98 \mathrm{~mm}$ OD, $18 \mathrm{~mm}$ height), and cooling coil made of copper. The air is injected from the bottom perforated plate to the upper liquid in the column forming bubbles, through the 83 holes (each $1 \mathrm{~mm}$ diameter) drilled in the perforated plate surface. The liquid in the bubble column dehumidifier is fresh water for the condensed vapor to be mixed with it and form fresh water production. 

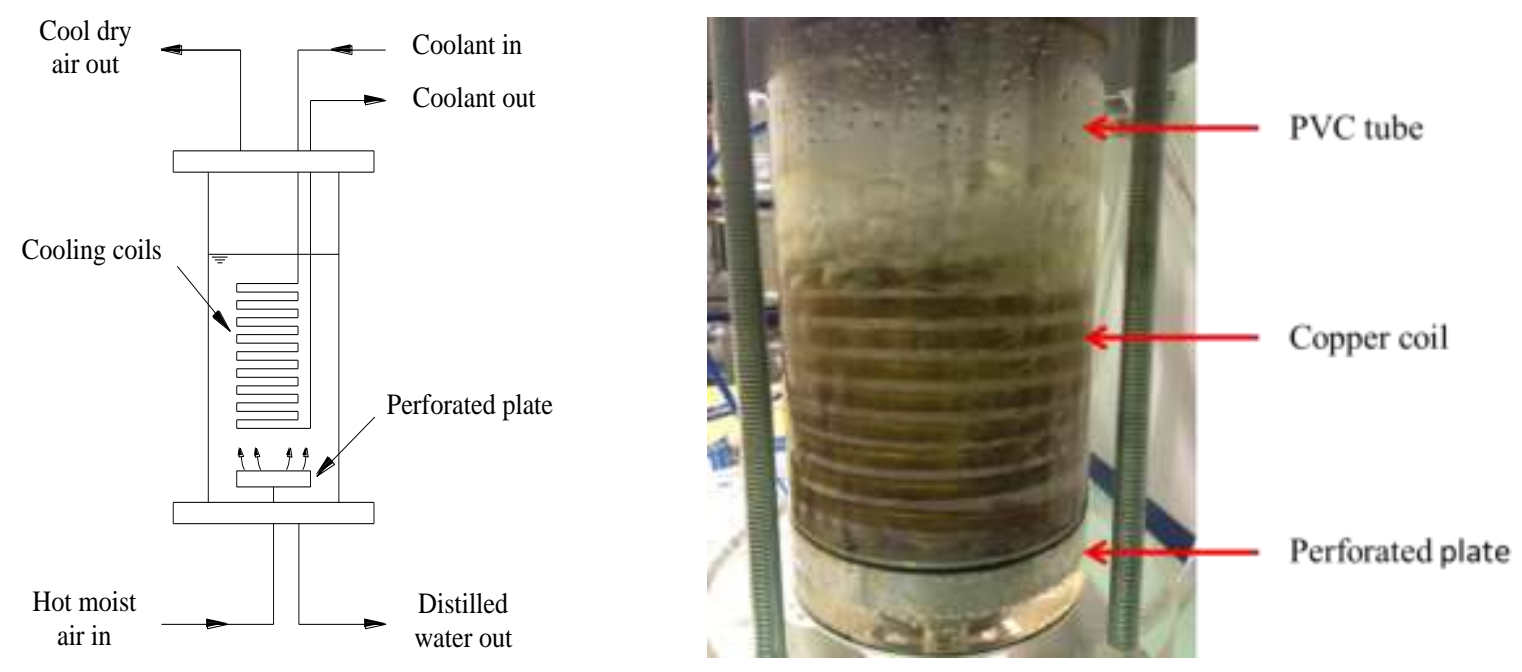

Fig. 1: Schematic and photograph of the bubble column reactor.

The experiments were conducted at four absolute pressures: $15 \mathrm{psi}(103.4 \mathrm{kPa}), 20 \mathrm{psi}(137.9 \mathrm{kPa}), 25 \mathrm{psi}(172.4 \mathrm{kPa})$ and $30 \mathrm{psi}(206.8 \mathrm{kPa})$. At each pressure set, the air flow was varied to produce a certain superficial velocity up to a maximum of $18 \mathrm{~cm} / \mathrm{s}$. The height of the liquid in the column was adjusted to 3,5 and $7 \mathrm{~cm}$ at each experimental setup. The inlet temperatures of the sparged humid air and the water in the cooling coil were fixed during the whole experiment, whereas the temperature of the water in the bubble column was left changeable by the operating conditions. All values of the experimental data were recorded when a steady state condition is approached.

The heat transfer rate is calculated using two ways: the heat released from the hot air as given by Eq. (1), and the heat gain by the cooling water as given by Eq. (2). The difference between the two values represents the heat losses from the apparatus which was less than $10 \%$ in all experiments.

$$
\begin{gathered}
\dot{q}_{a}=\dot{m}_{a}\left(i_{a i}-i_{a o}\right) \\
\dot{q}_{w}=\dot{m}_{w}\left(i_{w o}-i_{w i}\right)
\end{gathered}
$$

The enthalpies of the air and water are calculated using the Engineering Equation Solver (EES) software, where numerous physical properties of fluids are provided in the data package. The inlet and outlet air temperatures are measured using K-type thermocouples and it was assumed that the air is fully saturated at both ends of the bubble column. Similarly, the inlet and outlet temperatures of the circulated water were measured using rugged pipe plug K-type thermocouple probes and the inlet temperature was controlled using a constant temperature bath with an adjustable range of $0-80{ }^{\circ} \mathrm{C}$. The flow rates of air and water were measured by two rotameters installed in the apparatus: the former has a range of $0.5-4.5 \mathrm{ft}^{3} / \mathrm{min}$ $\left(236.0-2123.8 \mathrm{~cm}^{3} / \mathrm{s}\right)$, and the latter has a range of $0.8-8.0 \mathrm{~L} / \mathrm{min}\left(13.3-133.3 \mathrm{~cm}^{3} / \mathrm{s}\right)$.

The thermal effectiveness of the reactor was measured in this study. The effectiveness $(\varepsilon)$ is defined as the ratio of the actual enthalpy change to the maximum possible enthalpy change as given by Eq. (3). The maximum enthalpy change occurs for the fluid with minimum heat capacity rate which may be the air or the water inside the coil depending on the flow rate and the specific heat. As for this system, the maximum outlet temperature of either the air or the water occurs ideally equivalent to the temperature of the liquid in the column.

$$
\varepsilon=\frac{\Delta i_{a c t}}{\Delta i_{\max }}
$$




\section{Results and Discussion}

The effect of the water height in the bubble column on the total heat transfer rate and the effectiveness is shown in Fig. 2 and Fig. 3 respectively. It is found that the heat transfer rate and the effectiveness are nearly independent of the water height at the measured superficial velocities. These finding agrees well with previous experimental studies $[3,4]$. Therefore, we will present only experimental data at $5 \mathrm{~cm}$ water height in the following discussion.

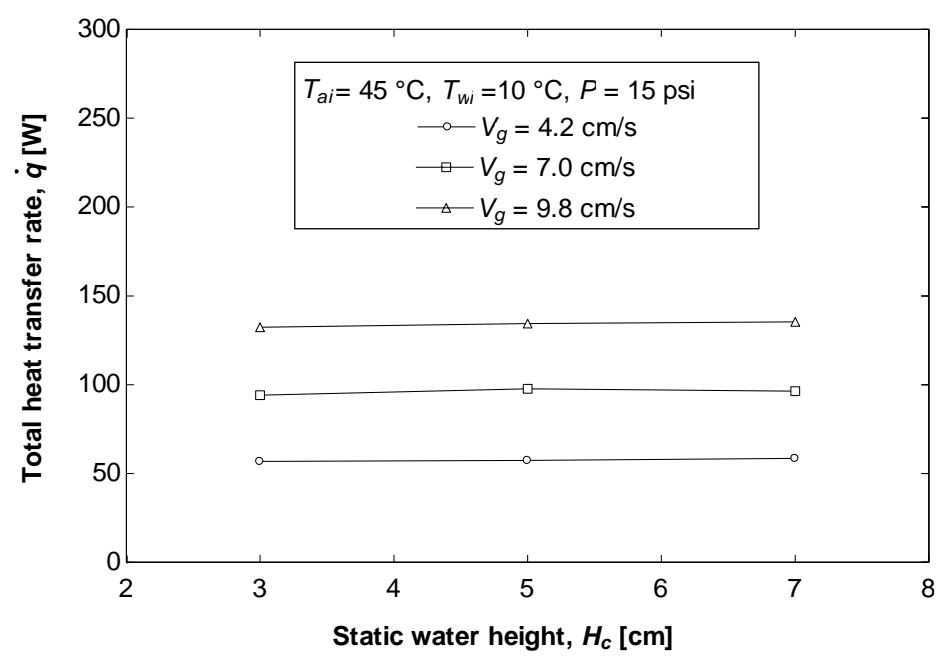

Fig. 2: Effect of the water height in the bubble column on the total heat transfer rate.

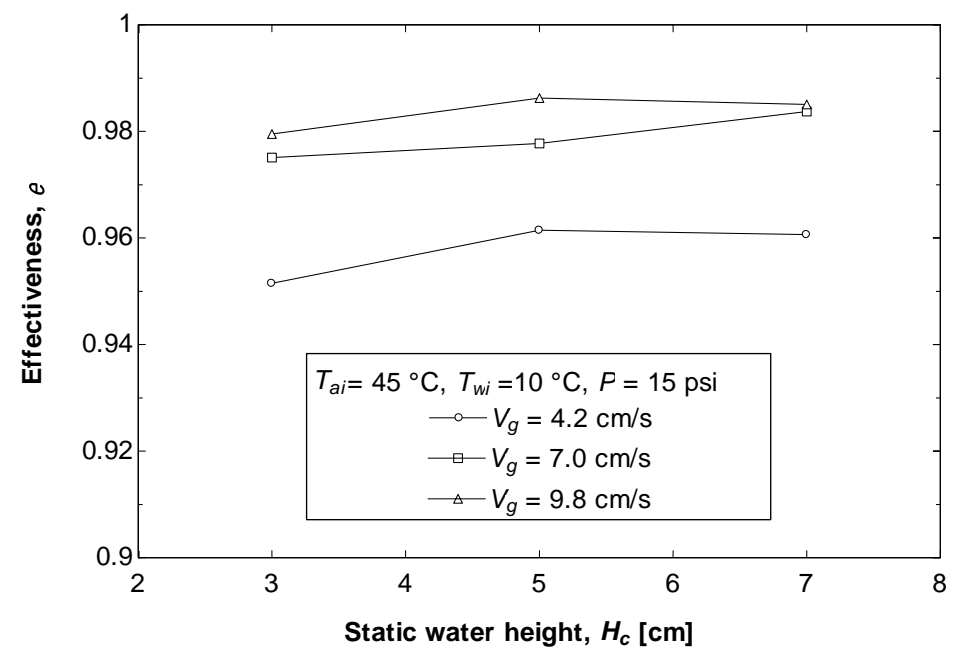

Fig. 3: Effect of the bubble column water height on the effectiveness.

The effect of the superficial velocity and pressure on the total heat transfer rate is illustrated in Fig. 4. The total heat transfer rate increases linearly with the superficial velocity as shown in the figure. This is due to the direct proportion with the air mass flow rate in the definition of the heat transfer rate. In addition, the increasing turbulence leads to the increase of the heat transfer coefficient and gas holdup. This is consistent with other relevant investigations $[3,4]$. The elevated pressure is found to slightly increase the total heat transfer rate since it directly causes an increase in the gas density resulting in an unstable disturbance and bubble breakup which both enhance the heat and mass transfer process. Moreover, the bubble size and bubble rise velocity are decreased which contribute to the increase of heat transfer coefficient corresponding to the increasing pressure too. 


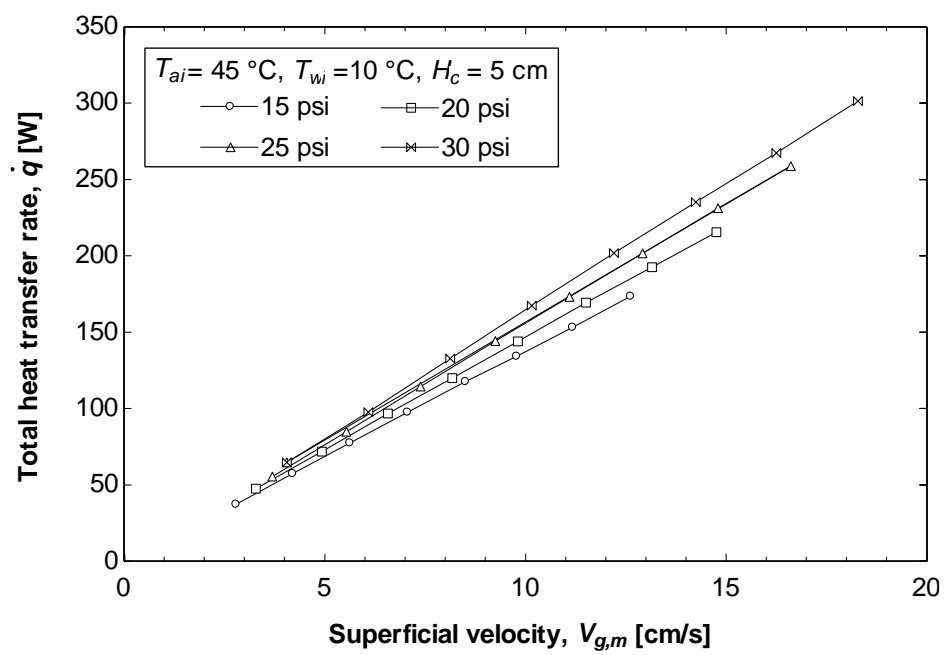

Fig. 4: Effects of the superficial velocity and the pressure on the total heat transfer rate.

The effect of varying superficial velocity and pressure on the effectiveness of the bubble column dehumidifier is shown in Fig. 5. The measurements indicate that the effectiveness increases significantly with increasing the superficial velocity at lower velocity values, while it approaches an asymptotic value at higher superficial velocity. On the other hand, the effectiveness decreases slightly at the increasing pressure. Learning from the definition of the effectiveness given by Eq. (3), the variation of the effectiveness (calculated on the air side) is associated with the variation of the enthalpies of exit air and liquid in the column at a given inlet air condition. Since the temperature of the liquid water in the column increases with the increase of the superficial velocity and pressure, the heat transfer rate increases too in that case. If the enthalpy or the temperature of the exit air increases faster than that of the liquid water in the column, the effectiveness would decrease as a consequence, otherwise the effectiveness should be increased.

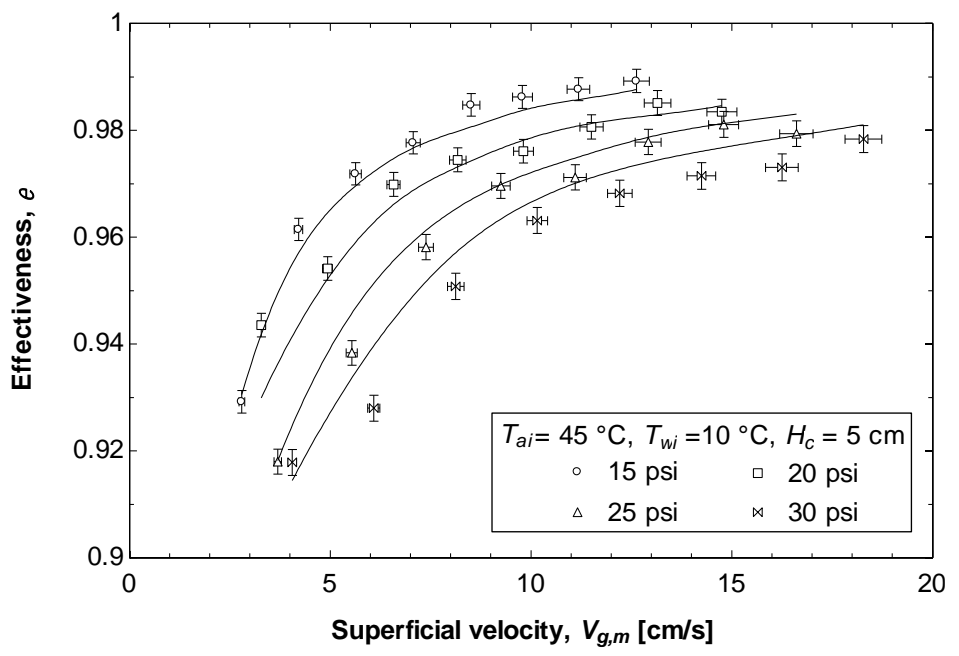

Fig. 5: Effects of the superficial velocity and the pressure on the effectiveness.

\section{Conclusion}

The experimental performance of a bubble column dehumidifier was investigated using a laboratory scale apparatus under varying operating conditions. The effects of the pressure, superficial velocity, and water column height on the performance of the bubble column dehumidifier are measured. It is concluded that the superficial velocity has a significant positive effect on both the heat transfer rate and the effectiveness. The elevated pressure increases the heat transfer rate it decreases the effectiveness. It is concluded that using a bubble column dehumidifier working at higher pressure for an HDH 
desalination system may increase its performance. However, it requires a larger bubble column dehumidifier (and higher cost) to compensate the decrease occurs in its effectiveness at higher pressure.

\section{References}

[1] S. A. El-Agouz, "Desalination based on humidification-dehumidification by air bubbles passing through brackish water," Chem. Eng. J., vol. 165, pp. 413-419, 2010.

[2] S. A. El-Agouz, M. Abugderah, "Experimental analysis of humidification process by air passing through seawater," Energy Convers. Manag., vol. 49, pp. 3698-3703, 2008

[3] G. P. Narayan, M. H. Sharqawy, S. Lam, S. K. Das, J. H. Lienhard V, "Bubble columns for condensation at high concentrations of noncondensable gas: Heat-transfer model and experiments," AIChE J. vol. 59, pp. 1780-1790, 2013.

[4] E. W. Tow, J. H. Lienhard V, "Experiments and modeling of bubble column dehumidifier performance," Int. J. Therm. Sci., vol. 80, pp. 65-75, 2014.

[5] A. E. Kabeel, M. H. Hamed, Z. M. Omara, S. W. Sharshir, "Water Desalination using a humidificationdehumidification technique - a detailed review," Nat. Resour., vol. 4, pp. 286-305, 2013.

[6] P. M. Wilkinson, H. Haringa, L. L. Van Dierendonck, "Mass transfer and bubble size in a bubble column under pressure," Chem. Eng. Sci., vol. 49, pp. 1417-1427, 1994.

[7] H. M. Letzel, J. C. Schouten, R. Krishna, C. M. van den Bleek, "Gas holdup and mass transfer in bubble column reactors operated at elevated pressure," Chem. Eng. Sci., vol. 54, pp. 2237-2246, 1999.

[8] N. Kantarci, F. Borak, K. O. Ulgen, "Bubble column reactors," Process Biochem., vol. 40, pp. 2263-2283, 2005.

[9] P. M. Wilkinson, L. L. v. Dierendonck, "Pressure and gas density effects on bubble break-up and gas hold-up in bubble columns," Chem. Eng. Sci. vol. 45, pp. 2309-2315, 1990.

[10] T.-J. Lin, L.-S. Fan, "Heat transfer and bubble characteristics from a nozzle in high-pressure bubble columns," Chem. Eng. Sci., vol. 54, pp. 4853-4859, 1999.

[11] T.-J. Lin, K. Tsuchiya, L.-S. Fan, "Bubble flow characteristics in bubble columns at elevated pressure and temperature," AIChE J., vol. 44, pp. 545-560, 1998.

[12] G. P. Narayan, R. K. McGovern, J. H. Lienhard, S. M. Zubair, "Variable pressure humidification dehumidification desalination system," in Proceedings of ASME/JSME Thermal Engineering Joint Conference, Honolulu, Hawaii, USA, 2011.

[13] H. Liu and M. H. Sharqawy, "Experimental performance of bubble column humidifier and dehumidifier under varying pressure," Int. J. Heat Mass Transf., vol. 93, pp. 934-944, 2016.

[14] H. Sadighi Dizaji, S. Jafarmadar, M. Abbasalizadeh, and S. Khorasani, "Experiments on air bubbles injection into a vertical shell and coiled tube heat exchanger; exergy and NTU analysis," Energy Convers. Manag., vol. 103, pp. 973-980, 2015. 